\title{
A Cross-layer Approach of Link Adaptation in IEEE 802.16 Broadband Wireless Access Systems ${ }^{1}$
}

\author{
Yu-Ting Yu and Hsi-Lu Chao \\ Department of Computer Science \\ National Chiao Tung University \\ hlchao@cs.nctu.edu.tw
}

\begin{abstract}
IEEE 802.16 Broadband Wireless Access system (BWA) is a promising emerging technology. In the IEEE 802.16 standard, multiple data transmission rates are defined by employing different modulation and channel coding schemes. How to select a proper mode affects the system performance significantly. In this paper, an efficient link adaptation scheme with QoS support is proposed. The proposed scheme takes both QoS demand and channel condition into consideration to determine a proper transmission mode. The QoS demand of a connection is represented as an expected packet error rate (PER). Based on this expected PER, the BS calculates the required signal-to-noise ratio (SNR) threshold of each mode. By comparing the measured SNR and all modes' SNR thresholds, the BS then suggests the SS a proper transmission mode. We evaluate the proposed scheme by simulations. The simulation results show that the link adaptation scheme improves the average system throughput and guarantees each connection's QoS demand.
\end{abstract}

Keywords: IEEE 802.16, link adaptation, PER, SNR, QoS

\section{Introduction}

The recent explosive growth of the Internet raises the demands for higher capacity, higher data rate, and more advanced multimedia services to customers. The IEEE 802.16 standard is designed to support these demands and specifies the WirelessMAX ${ }^{\mathrm{TM}}$ air interface [1]. The IEEE 802.16 BWA systems have many advantages, such as rapid deployment, high speed data rate, high scalability, multimedia services, and lower maintenance, and upgrade costs.

The standard addresses systems that operate from $10 \mathrm{GHz}$ to $66 \mathrm{GHz}$ and the physical layers (PHYs) provide multiple data transmission rates by employing different modulation and channel coding schemes. The mechanism to select one out of multiple available transmission rates is referred to as link adaptation and the effectiveness of the implemented link adaptation scheme can affect the system performance significantly. IEEE 802.16 does define a radio link control (RLC) framework to enable the implementation of PHY layer adaptation schemes. This framework includes the message definitions and signal flow for link adaptation. Unfortunately, it does not define a detailed link adaptation algorithm.

The goal of this paper is to propose an efficient link adaptation scheme for IEEE 802.16 BWA systems, which is based on the perspectives of PHY and network layers. Specifically, an application can be assigned a packet error rate (PER) to indicate its QoS demand. Based on the PER, adopted channel error model, and measured signal-to-noise ratio (SNR), the base station (BS) suggests the subscriber station (SS) a proper transmission PHY mode. The objectives of the proposed link adaptation scheme are system throughput improvement and QoS guarantee.

The rest of this paper is organized as follows. Section II introduces the related work. The proposed link adaptation scheme is described in Section III. Section IV presents and discusses the simulation results. Finally, this paper concludes with Section V.

\section{Related Work}

In [2], a TCP delay-based LRA algorithm for IEEE 802.16-2004 was proposed. Through experiment results, [2] derives the SNR switching thresholds for acceptable end-toend TCP delay of QPSK, 16-QAM, and 64-QAM modulation schemes. An SS selects the best modulation scheme according to the measured SNR value. The drawback of [2] is that it ignores the effect of FEC mechanism. Upon different RS code and $\mathrm{CC}$ code, the selected modulation scheme may be mapped to different PHY mode. Besides, the corresponding end-to-end TCP delay for a specific SNR value was obtained via a simple scenario, i.e., only one FTP flow between an SS and a BS. This scenario did not take other flows' impact into consideration. Therefore, the suggested switching thresholds may be with error.

$[3,4]$ focused on the performance evaluation of an IEEE 802.16-2004 based system in jamming environment. The simulation results showed that under the same jamming

\footnotetext{
${ }^{1}$ This work was supported in part by National Science Council under grant NSC95-2219-E-009-014-, in part by the National Science Council under grant NSC-95-2221-E-009-019, and in part by the Intel Research Council.
} 


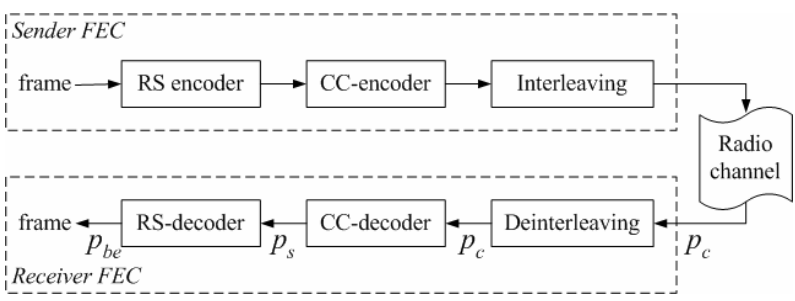

Figure 1 The block diagram of FEC operations

power, the system throughput of multitone pilot jamming degrades more seriously than that of partial-band jamming. Besides, a mean carrier-to-interference-noise ratio (CINR)based link rate adaptation scheme was proposed in [3, 4]. When the updated mean CINR is out of the required range of the current PHY mode, the mode will be upgraded or downgraded accordingly. Since $[4,5]$ used a mean CINR estimator of Exponentially Weighted Moving Average (EWMA) to smoothen the estimated values, the selected and theoretical PHY modes may not be identical (for example, high variance of channel condition).

\section{The Proposed Link Adaptation Scheme}

The key design issue for a link adaptation scheme is to choose a proper metric to select the transmission PHY mode. Our proposed link adaptation scheme adopts a concept of cross-layer design to achieve mode selection and QoS support simultaneously. Specifically, based on the expected PER of a connection and the adopted channel model, the BS derives the acceptable SNR range of each PHY mode (described in Section 3.1.). Furthermore, the BS suggests the SS a proper transmission PHY mode, based on the measured SNR value (described in Section 3.2.). Both phases (i.e., PHY mode selection and acceptable SNR range derivation) are described as follows.

\subsection{Acceptable SNR range derivation}

In this paper, we focus on WirelessMAN OFDM-PHY that is based on Orthogonal Frequency Division Multiplexing (OFDM) modulation and designed for non-line-of-sight (NLOS) operation in the frequency bands below $11 \mathrm{GHz}$. There are seven mandatory PHY modes with various modulation schemes and coding rates defined in OFDMPHY. Details of these PHY modes are listed in Table I.

In OFDM-PHY, the Forward Error Control (FEC) consists of a Reed-Solomon (RS) outer code and a rate-compatible convolutional inner code. The block diagram of FEC operations is shown in Fig. 1.

In order to derive the acceptable SNR ranges of each PHY mode, we assume fixed packet size and each is with size $B$. Therefore, given a specific PER, the corresponding bit error rate $P_{b e}$ is

$$
P_{b e}=1-\sqrt[B]{(1-P E R)}
$$

Table I Seven PHY modes of IEEE 802.16 OFDM PHY

\begin{tabular}{|c|c|c|c|c|c|c|}
\hline Mode & $\begin{array}{c}\text { Data rate } \\
\text { (Mbps) }\end{array}$ & Modulation & $\begin{array}{c}\text { Bytes per } \\
\text { symbol }\end{array}$ & $\begin{array}{c}\text { Overall } \\
\text { code rate }\end{array}$ & $\begin{array}{c}\text { RS code } \\
(\mathbf{N}, \mathbf{K}, \mathbf{T})\end{array}$ & $\begin{array}{c}\text { CC code } \\
\text { rate }\end{array}$ \\
\hline 1 & 3.9544 & BPSK & 12 & $1 / 2$ & $(12,12,0)$ & $1 / 2$ \\
\hline 2 & 7.9096 & QPSK & 24 & $1 / 2$ & $(32,24,4)$ & $2 / 3$ \\
\hline 3 & 11.8648 & QPSK & 36 & $3 / 4$ & $(40,36,2)$ & $5 / 6$ \\
\hline 4 & 15.82 & 16-QAM & 48 & $1 / 2$ & $(64,48,8)$ & $2 / 3$ \\
\hline 5 & 23.7304 & 16-QAM & 72 & $3 / 4$ & $(80,72,4)$ & $5 / 6$ \\
\hline 6 & 31.6408 & 64-QAM & 96 & $2 / 3$ & $(108,96,6)$ & $3 / 4$ \\
\hline 7 & 35.596 & 64-QAM & 108 & $3 / 4$ & $(120,108,6)$ & $5 / 6$ \\
\hline
\end{tabular}

Furthermore, the $P_{b e}$ can be used to calculate the symbol error rate (i.e., $P_{s}$ shown in Fig. 1), as in (2).

$$
\sum_{i=T+1}^{n} i\left(\begin{array}{c}
n \\
i
\end{array}\right) P_{S}^{i}\left(1-P_{S}\right)^{n-i} \geq n P_{b e},
$$

where $T$ is the maximum number of bits that can be corrected in each RS code block, and $n$ is the number of bits in each block. In addition, the bit error rate (denoted as $P_{c b}$ ) before RS-decoding for symbol in $\mathrm{GF}\left(2^{b}\right)$ can be derived from $P_{s}$ by using (3).

$$
P_{c b} \geq \frac{1}{b} P_{s},
$$

where $b$ is a positive integer. In IEEE 802.16 OFDM-PHY, $b=8[1]$.

To derive the bit error rate before CC-decoding(i.e., $P_{c}$ shown in Fig 1), we need to further calculate the probability that an incorrect path at distance $k$ from the correct path is chosen by the Viterbi decoder, and this probability is denoted as $P_{k}$. From [5], $P_{k}$ is approximated as

$$
\sum_{k=d_{f}}^{d_{f}+N}\left(c_{k} \times P_{k}\right) \approx a P_{c b},
$$

where $a$ is the number of bits input into the CC-encoder, $d_{f}$ is the free distance of the convolutional code in a specific PHY mode, $c_{k}$ is the number of error bits that occur in all incorrect paths in the trellis that differ from the correct path in exactly $k$ positions. From [6], the bit error rate $p_{c}$ can be derived by using (5).

$$
p_{k}=\left\{\begin{array}{l}
\sum_{i=(k+1) / 2}^{k}\left(\begin{array}{l}
k \\
i
\end{array}\right) p_{c}^{i}\left(1-p_{c}\right)^{k-i}, \text { if } k \text { is odd } \\
\frac{1}{2}\left(\begin{array}{c}
k \\
k / 2
\end{array}\right) P_{c}^{k / 2}\left(1-P_{c}\right)^{k / 2}+\sum_{i=k / 2+1}^{k}\left(\begin{array}{c}
k \\
i
\end{array}\right) P_{c}^{i}\left(1-P_{c}\right)^{k-i}, \\
\text { is even }
\end{array}\right.
$$

Lastly, the $P_{c}$ can be inputted into the adopted channel error model to obtain the corresponding SNR (denoted as $\mathrm{E}_{\mathrm{b}} / \mathrm{N}_{0}$ ) operation threshold of each PHY mode.

\subsection{PHY mode selection}

The PHY mode selection is detailed as follows: 
(1) An SS informs its BS the expected PER of the new service flow $i$.

(2) Under the adopted channel error model and specified PER requirement, the BS then calculates the corresponding acceptable $\mathrm{E}_{\mathrm{b}} / \mathrm{N}_{0}$ ranges of all PHY modes.

(3) Comparing the measured $\mathrm{E}_{\mathrm{b}} / \mathrm{N}_{0}$ with the obtained SNR range of each PHY mode, the BS suggests the SS the best one, say mode $m$.

Based on the supported data rate of PHY mode $m$ (denoted as $R_{m}$ ) and that service flow $i$ 's queued data size, the BS calculates the number of slots the service flow $i$ needs to the $\mathrm{SS}$, as shown in (1).

$$
N u m_{-} \text {slot }[i]=\frac{\text { data_queued }[i]}{R_{m} \times T_{\text {slot }}},
$$

where $T_{\text {slot }}$ is the slot time. In order to suggest the SS changing the PHY mode timely, the BS keeps monitoring channel condition.

\subsection{An Example}

In the following, we use Additive White Gaussian Noise (AWGN) channel model as an example to show the obtained operation range of each PHY mode. The settings of N, K, and $\mathrm{T}$ values of RS coder are as listed in Table I. We first set PER be 0.1 . The required $E_{b} / N_{0}$ thresholds to satisfy PER = 0.1 QoS demand for seven modes are listed in Table II. Thus to have $\mathrm{PER}=0.1$ performance, an SS can operate at mode 2 only when the measured $E_{b} / N_{0}$ is larger than the threshold, i.e., $0.0863 \mathrm{~dB}$. It is interesting that mode 2 has a lower $\mathrm{E}_{\mathrm{b}} / \mathrm{N}_{0}$ threshold than that of mode 1 . Similarly, the required $E_{b} / N_{0}$ of mode 4 is smaller than that of mode 3 . One possible reason is that we use AWGN model, which ignores consider some interference and fading phenomenon, thus the bit error rates of modes 1 and 2 differ slightly. Moreover, the T value of RS code of mode 1 shown in Table I is zero, and this implies that no data byte can be corrected after RS-decoder. Therefore, mode 1 needs larger $\mathrm{E}_{\mathrm{b}} / \mathrm{N}_{0}$ to achieve the same PER. Similarly, the CC code rate of mode 3 is higher than that of mode 4, as shown in Table I. That means that mode 4 has better correction rate and require smaller $\mathrm{E}_{\mathrm{b}} / \mathrm{N}_{0}$ to have the same PER performance. Therefore, we need only five PHY modes, i.e., modes 2, 4, 5, 6, and 7, for the case of PER $=0.1$.

From Table II, we can further adopt $2.4481 \mathrm{~dB}, 7.0797 \mathrm{~dB}$, $8.9658 \mathrm{~dB}$, and $10.94335 \mathrm{~dB}$ as the boundaries to define acceptable $\mathrm{E}_{\mathrm{b}} / \mathrm{N}_{0}$ ranges of proper modes. The obtained acceptable $\mathrm{E}_{\mathrm{b}} / \mathrm{N}_{0}$ ranges are listed in Table III.

The simulated error performances for all PHY modes upon different PER setting are shown in Fig. 2. The $x$-axis and $y$ axis are the measured $\mathrm{E}_{\mathrm{b}} / \mathrm{N}_{0}$ and the PER, respectively. Again, modes 1 and 3 need higher Eb/N0 requirements for all PER settings, compared with modes 2 and 4 .
Table II $E_{b} / N_{0}$ threshold of each PHY mode upon PER $=10^{-1}$ in AWGN channel

\begin{tabular}{|c|c|c|c|c|c|c|c|}
\hline Mode & 1 & 2 & 3 & 4 & 5 & 6 & 7 \\
\hline$E_{b} / N_{0}(\mathrm{~dB})$ & 0.3535 & 0.0863 & 3.8109 & 2.4481 & 7.0797 & 8.9658 & 10.94335 \\
\hline
\end{tabular}

Table III Acceptable $\mathrm{E}_{\mathrm{b}} / \mathrm{N}_{0}$ range of each reduced mode upon PER $=10^{-1}$ in AWGN channel

\begin{tabular}{|c|c|c|c|c|c|}
\hline Mode & 2 & 4 & 5 & 6 & 7 \\
\hline$E_{b} / N_{o}(\mathrm{~dB})$ & {$[0,2.4481)$} & {$[2.4481$,} & {$[7.0791$,} & {$[8.9658$,} & {$[10.94335$,} \\
& $7.0797)$ & $8.9658)$ & $10.94335)$ & $\infty)$ \\
\hline
\end{tabular}

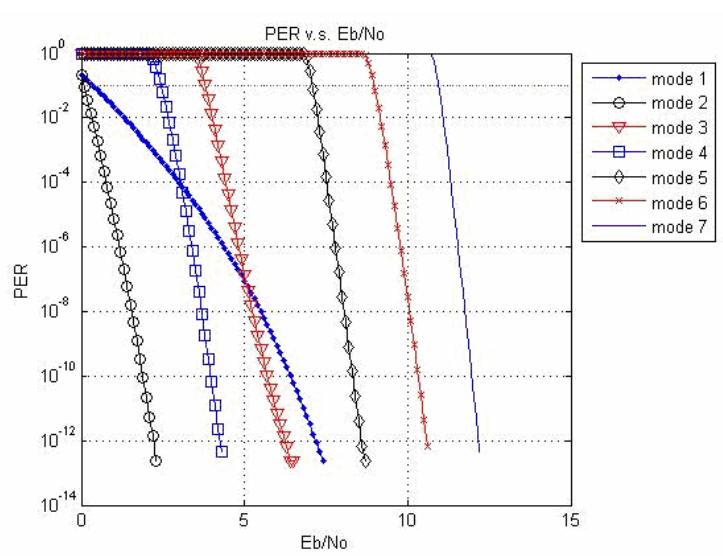

Figure 2 The error performance in the AWGN channel

\section{Performance Evaluation}

In this section, we evaluate our proposed link adaptation scheme (denoted as Q-LRA). We simulate 1000 frames, and each frame is with duration of $10 \mathrm{~ms}$. The control overheads for uplink (UL) and downlink (DL) sub-frames of each mode are based on the suggestions listed in [1], and the data rate of each mode are listed in Table I. The channel bandwidth is 10 $\mathrm{MHz}$ and each packet is with a fixed size of 256 bytes. We assume one BS and ten SSs in our simulations and generate 20, 30, and 40 connections (denoted as scenario 1, 2, 3), respectively. Each SS has at least one connection and each connection is randomly assigned a PER from $[0.05,0.1,0.15$, $0.2,0.25,0.3]$. The measured channel quality is uniformly distributed within $[0,30 \mathrm{~dB}]$. Again, we adopt AWGN as the channel model.

The performance metrics measured in the simulation include:

(1) Average system throughput $(\varphi)$ : defined as the ratio of the number of successfully transmitted data bits to the simulation time.

(2) Average satisfaction ratio $(\eta)$ : the percentage of connections whose QoS demands (i.e., PER) have been satisfied. Indeed, the PER of a connection is the ratio of the number of failed packets to the number of transmitted 
Table IV. Simulation results of average system throughput and average satisfaction ratio

\begin{tabular}{|c|c||c|c|c|c|c|c|c|c|}
\hline \multicolumn{2}{|c|}{$\begin{array}{c}\text { Scheme } \\
\text { Scenario }\end{array}$} & Q-LRA & Mode 1 & Mode 2 & Mode 3 & Mode 4 & Mode 5 & Mode 6 & Mode 7 \\
\hline \hline \multirow{2}{*}{$\mathbf{1}$} & $\varphi$ & 24.4660 & 2.6483 & 5.2602 & 7.5760 & 10.1476 & 12.7935 & 16.9060 & 17.5557 \\
\cline { 2 - 10 } & $\eta$ & 1.0000 & 0.6313 & 0.6313 & 0.6125 & 0.6125 & 0.5188 & 0.5188 & 0.4688 \\
\hline \multirow{2}{*}{$\mathbf{2}$} & $\varphi$ & 24.2739 & 2.8629 & 5.6305 & 7.8652 & 10.8012 & 12.7924 & 16.3161 & 18.0436 \\
\cline { 2 - 10 } & $\eta$ & 1.0000 & 0.6704 & 0.6704 & 0.6148 & 0.6370 & 0.5037 & 0.4852 & 0.4741 \\
\hline \multirow{2}{*}{3} & $\varphi$ & 24.2680 & 2.9810 & 6.0126 & 8.3522 & 11.6148 & 15.4952 & 19.9781 & 21.4400 \\
\cline { 2 - 9 } & $\eta$ & 1.0000 & 0.7350 & 0.7525 & 0.6900 & 0.7175 & 0.6450 & 0.6150 & 0.5900 \\
\hline
\end{tabular}

packets.

The simulation results for average system throughput and average satisfaction ratio are summarized in Table IV, where mode $i$ scheme $(\mathrm{i}=1,2, . ., 7)$ indicates the SS always operates at PHY mode $i$, no matter what channel quality is. We found that Q-LRA has the largest throughput, followed by modes 7, $6,5,4,3,2$, and 1 . For satisfaction ratio, the proposed scheme (i.e., Q-LRA) outperforms others (with $\eta=1.0000$ for all scenarios). However, modes 1 and 2 have similar satisfaction ratio; modes 3 and 4 have similar satisfaction ratio. It is interesting that Q-LRA performs better than mode 7 in satisfaction ratio. To illustrate the reason clearly, we show the data rates and $E_{b} / N_{0}$ values of the first 70 -ms of the third scenario in Figs. 3(a) and 3(b). In Fig. 3(a), the blue curve represents the Q-LRA performance. The key characteristic of Q-LRA is that an SS will update its PHY mode when channel quality changes. Thus at about $20-\mathrm{ms}$, the channel quality gets worse from $17.4 \mathrm{~dB}$ to $2.7 \mathrm{~dB}$ (see Fig. 3(b)), and the SS changes the transmission PHY mode from mode 7 to mode 2 . Since the measured channel quality $(2.7 \mathrm{~dB})$ is much lower than the $\mathrm{E}_{\mathrm{b}} / \mathrm{N}_{0}$ threshold of mode 7 , the scheme of always-operating-at-mode- 7 incurs lots of transmission failures for about $4.5 \mathrm{~ms}$, and thus has low average throughput and low satisfaction ratio. When channel condition recovers, the SS selects mode 7 again. Thus QLRA performs better, compared with other fixed-mode schemes. Note that the BS suggests an SS a proper mode through a connection's PER, the proposed scheme can guarantee connections' QoS demands.

\section{Conclusions}

In this paper, we have proposed an efficient link adaptation algorithm with QoS support for a BS to suggest an SS a proper PHY mode. Instead of only considering channel quality, our proposed scheme takes both QoS demand and channel condition into consideration to make suggestion. We use PER as the indicator of a connection's

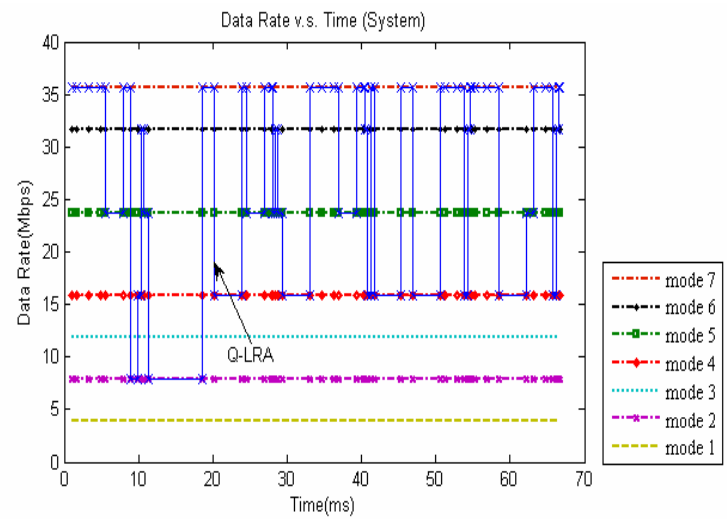

(a) Adopted data rate for all schemes

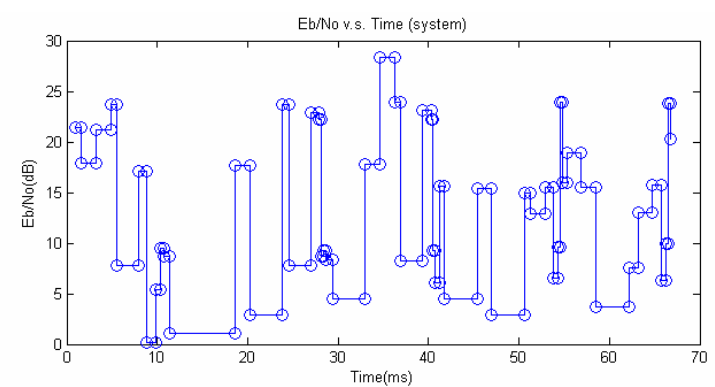

(b) Measured $\mathrm{E}_{\mathrm{b}} / \mathrm{N}_{\mathrm{o}}$ vs. time

Figure 3 The first 70-ms simulation result

QoS demand. Based on the specified PER, the BS calculates the required $\mathrm{E}_{\mathrm{b}} / \mathrm{N}_{0}$ threshold of each mode, and suggests the SS which PHY mode to operate at. The simulation results show that our proposed scheme improves the average system throughput and guarantees each connection's QoS demand.

\section{References}

[1] IEEE 802.16-REVd/D5-2004, "IEEE Standard for Local and Metropolitan Area Networks-Part 16: Air Interface for Fixed Broadband Wireless Access Systems," May 13, 2004.

[2] S. Ramachandran, C. W. Bostian, and S. F. Midkiff, "A Link Adaptation Algorithm for IEEE 802.16," Proc. IEEE WCNC 2005 , pp. 1466-1471.

[3] Nai'an Liu, Dechun Sun, Wuqiang Li, and Changxing Pei, "A link adaptation solution to IEEE 802.16d WMAN," Proc. IEEE PDCAT 2006.

[4] Juan Li and Sven-Gustav Haggman, "Performance of IEEE802.16-2004 based system in jamming environment and its improvement with link adaptation," Proc. IEEE PIMRC 2006.

[5] $\mathrm{P}^{\circ}$ al Frenger, $\mathrm{P}^{\circ}$ al Orten, and Tony Ottosson, "Convolutional Codes with Optimum Distance Spectrum," IEEE Communications Letters, Vol. 3, No. 11, pp.317-319, Nov. 1999 , pp. 317-319.

[6] Greg M. Chiasson and Michael B. Pursley, "Concatenated coding for frequency-hop packet radio," IEEE Military Communications Conference, Nov. 1991, pp. 1235-1239. 